(2) that the form of variation among the units is approximately normal;

(3) that the sample is drawn from a supply with at least twenty times as many units as are in the sample.

The basis of control charts is briefly described, with special reference to the methods of estimating the standard deviation of the population. It seems to the reviewer that this publication adapts recent progress in sampling theory admirably to a wide range of applied problems in quality control.

\title{
H. L. RIETZ
}

Einführung in die Liniengeometrie und Kinematik. By Ernst August Weiss. Berlin and Leipzig, Teubner, 1935. vi+122 pp.

This is volume 41 of Teubner's Mathematische Leitfäden and is written by Professor Weiss of the University of Bonn, a pupil of E. Study, the notable geometrician who made the same university a well known center of mathematical activity. The contents of the little text, as Weiss states, are closely connected with the name of Study and it is one of the author's aims to introduce the reader to Study's methods.

After discussion of the linear complex in $R_{3}$, line space is mapped upon $R_{5}$ as first conceived by Klein. Then follows an investigation of manifolds of complexes and the loci of points and planes connected with them; next an account of Lie's line-sphere transformation and applications in kinematics. The principal theorems of movements and reflexions are derived by the elegant calculus of biquaternions. An important feature of the book is the use of complex spaces and hyperspaces.

The whole treatment is very interesting and affords the reader an insight into a number of novel ideas, which may act as stimulants for workers in this field. The reviewer, however, is under the impression that for the purpose in view and the results obtained, the analytical machinery is in places unnecessarily artificial.

Arnold EMch

Arithmétique et Géométrie sur les Variétés Algébriques. By André Weil. 16 pp. Quelques Propriétés des Variétés Algébriques se Rattachant aux Théories de l'Algébre Moderne. By Paul Dubreil. Actualités Scientifiques et Industrielles, Nos. 206, 210. Exposés mathématiques publiés à la mémoire de Jacques Herbrand, Nos. XI, XII. Paris, Hermann, 1935. 33 pp.

These two booklets belong to a series of memoirs published by friends to commemorate Jacques Herbrand, the young French mathematician whose premature death was a severe loss for modern French mathematics. The present pamphlets both deal with the general polynomial ideal theory of $\mathrm{E}$. Noether and van der Waerden, and its applications to algebraic problems. Weil continues investigations by Siegel and himself on the general theory of diophantine equations and shows how certain algebraic decomposition theorems correspond to number theoretical decompositions. Dubreil first studies some properties of the decomposition of homogeneous ideals into primary components and these results are then used to derive general theorems on algebraic varieties.

Oystein Ore 